\title{
A Census of Small Latin Hypercubes
}

\author{
Brendan D. McKay \\ Department of Computer Science \\ Australian National University \\ Canberra, ACT 0200, Australia \\ bdm@cs.anu.edu.au
}

\author{
Ian. M. Wanless \\ School of Mathematical Sciences \\ Monash University \\ Clayton, Vic 3800, Australia \\ ian.wanless@sci.monash.edu.au
}

\begin{abstract}
We count all latin cubes of order $n \leq 6$ and latin hypercubes of order $n \leq 5$ and dimension $d \leq 5$. We classify these (hyper)cubes into isotopy classes and paratopy classes (main classes). For the same values of $n$ and $d$ we classify all $d$-ary quasigroups of order $n$ into isomorphism classes and also count them according to the number of identity elements they possess (meaning we have counted the $d$-ary loops).

We also give an exact formula for the number of (isomorphism classes of) $d$-ary quasigroups of order 3 for every $d$. Then we give a number of constructions for $d$-ary quasigroups with a specific number of identity elements. In the process, we prove that no 3 -ary loop of order $n$ can have exactly $n-1$ identity elements (but no such result holds in dimensions other than 3). Finally, we give some new examples of latin cuboids which cannot be extended to latin cubes.
\end{abstract}

\section{Basic definitions}

Let $[n]$ denote the set $\{1,2, \ldots, n\}$ and let $[n]^{d}$ denote the cartesian product $[n] \times[n] \times$ $\cdots \times[n]$ of $d$ copies of $[n]$. By a hypercube of order $n$ and dimension $d$ we mean a $d$ dimensional array of $n^{d}$ cells where the cells are indexed by $[n]^{d}$ and each cell contains an element of $[n]$ (which we will call a symbol). Suppose that $H$ is such a hypercube and $c$ is any cell of $H$. A line through $c$ is the set of $n$ cells of $H$ whose coordinates match those of $c$ except possibly in the $k$-th coordinate (there is one line for each choice of $k$ ). A hyperplane through $c$ is the set of $n^{d-1}$ cells of $H$ whose $k$-th coordinate matches that of $c$ (there is one hyperplane for each choice of $k$ ). Any hyperplane in a $d$-dimensional hypercube can be considered to be a $(d-1)$-dimensional hypercube, simply by dropping the common coordinate. We use vector notation such as $\vec{v}$ for an element of $[n]^{d}$. In a 
hypercube $H$ we denote the symbol in the cell with coordinates $\vec{v}=\left(v_{1}, v_{2}, \ldots, v_{d}\right)$ by $H(\vec{v})$ or $H\left(v_{1}, v_{2}, \ldots, v_{d}\right)$.

We say that a hypercube $H$ is latin if the $n$ cells in any line of $H$ contain each of the symbols in $[n]$ exactly once. We define $\mathcal{H}_{n}^{d}$ to be the set of latin hypercubes of order $n$ and dimension $d$. Given a hypercube $L \in \mathcal{H}_{n}^{d}$ and a cell $\vec{v}=\left(v_{1}, v_{2}, \ldots, v_{d}\right) \in[n]^{d}$, we can define $d$ permutations of $[n]$ corresponding to the lines through $\vec{v}$. Namely, for each $j \in[d]$ we define $\rho_{j}(\vec{v}, L)$ to be the permutation

$$
k \mapsto L\left(i_{1}, \ldots, i_{j-1}, k, i_{j+1}, \ldots, i_{d}\right) \quad \text { for } k \in[n] .
$$

If $\rho_{j}((1,1, \ldots, 1), L)$ is the identity permutation for each $j \in[d]$ then we say that $L$ is reduced. We define $\mathcal{R}_{n}^{d} \subseteq \mathcal{H}_{n}^{d}$ to be the set of reduced latin hypercubes of order $n$ and dimension $d$.

For some purposes it is easier to think of each hypercube $H \in \mathcal{H}_{n}^{d}$ as, instead, a set $T_{H} \subseteq[n]^{d+1}$ where for each $\vec{v}=\left(v_{1}, v_{2}, \ldots, v_{d}\right) \in[n]^{d}$ there is a $(d+1)$-tuple $\left\langle v_{1}, v_{2}, \ldots, v_{d}, H(\vec{v})\right\rangle$ in $T_{H}$. (We adopt the convention of writing the tuples of a hypercube inside angle brackets \langle\rangle .) The latin property of the hypercube is equivalent to saying that no two of its tuples differ in exactly one coordinate.

Some of the interest in latin hypercubes stems from coding theory, given that a hypercube $L \in \mathcal{H}_{n}^{d}$ is equivalent to a maximum distance separable (MDS) code over an alphabet of size $n$, with length $d+1$ and minimum distance 2. See Laywine and Mullen [16] for a good introduction to this area.

Another source of interest in latin hypercubes is in non-associative algebra. A d-ary quasigroup of order $n$ is a function $Q:[n]^{d} \rightarrow[n]$ such that $Q(\vec{u}) \neq Q(\vec{v})$ whenever $\vec{u}$ and $\vec{v}$ differ in exactly one coordinate. Such a quasigroup is clearly equivalent to a latin hypercube $L \in \mathcal{H}_{n}^{d}$ where $L(\vec{v})=Q(\vec{v})$. Moreover $L$ is reduced if and only if 1 is an identity element in the corresponding quasigroup $Q$. (An identity element of $Q$ is an $x \in Q$ such that

$$
Q(y, x, x, \ldots, x)=Q(x, y, x, \ldots, x)=\cdots=Q(x, x, \ldots, x, y)=y
$$

for all $y \in Q$.) A quasigroup possessing an identity element is called a loop. One of the aims of this paper is to count $d$-ary quasigroups and $d$-ary loops of order $n$ (up to isomorphism) for small $d$ and $n$. We do this using the theory in Section 2 and the algorithm in Section 3. For $d>2$ it transpires that identity elements in $d$-ary loops need not be unique. Hence, in Section 5 we examine constructions for loops with various numbers of identity elements.

A third source of applications for latin hypercubes is in the design of statistical experiments. In this field the phrases "latin cube" and "latin hypercube" are used for a broader class of objects than we are allowing in our definition. See [6] or [24] in this regard. The statistical definition of latin hypercube seems unnatural in other contexts since it does 
not have the property that each hyperplane is necessarily a latin hypercube of one lower dimension. The confusion arising from these different definitions is unfortunate, but we choose to follow, for example [4] and [15] in our use of "latin hypercube" and many papers (e.g. $[8,9,14,17,22])$ in our use of "latin cube". The alternative terminology "permutation (hyper)cube" was advocated in [6] and used in [1,3,7,11]. This terminology is better reserved for the higher dimensional analogues of permutation matrices (that is, multi-dimensional arrays with all entries 0 or 1 such that each line contains a unique 1 ). Interestingly, $(d+1)$-dimensional permutation hypercubes of order $n$ are combinatorial objects essentially equivalent to $d$-dimensional latin hypercubes of order $n$ (both objects described in our preferred terminology). This simple observation was made by Gupta [9], who used the phrase "permutation cube" in our preferred sense.

In the cases $d=1,2,3$ a latin hypercube of dimension $d$ is a permutation, a latin square and a latin cube, respectively. Much of the theory of latin hypercubes is yet to be developed although some analogues of results for latin squares have been investigated for latin cubes and occasionally for hypercubes in general. Completion and embedding results have been obtained by Cruse [4], Kochol [14] and Lindner [17]. Fu [8] considered the range of values possible for the number of common entries shared by distinct latin cubes (in other terminology, this equates to studying the possible sizes of trades in latin cubes). A number of authors have considered sets of $k$ mutually orthogonal latin hypercubes. Here again several different definitions are possible; see [6] and [16] for further information and references. The notions of intercalates, transversals and prolongation were generalised by Heinrich [11] from latin squares to higher dimensions. The same generalisation of transversals was used in [15] but a different generalisation of transversals was given by Beljavskaja and Murathudjaev [3]. Finally, strongly diagonal latin squares (also called Knut Vik designs) and totally symmetric latin squares were generalised to higher dimensions by Alavi et al. [1] and Bailey et al. [2] respectively.

The usual notions of isotopism, paratopism and isomorphism generalise naturally from latin squares to higher dimension. Let $S_{n}$ be the symmetric group on $[n]$ and let $S_{n}^{c}$ denote the direct product of $c$ copies of $S_{n}$. Then the natural action of $S_{n}^{d+1}$ on $[n]^{d+1}$ induces an action on $\mathcal{H}_{n}^{d}$ (where, as discussed above, we associate each $H \in \mathcal{H}_{n}^{d}$ with a subset $T_{H} \subseteq[n]^{d+1}$ ). This action is called isotopism (or isotopy) and its orbits are called isotopy classes. Define $\Delta_{n}^{d+1}$ to be the diagonal subgroup of $S_{n}^{d+1}$, that is $\Delta_{n}^{d+1}=\{(g, g, \ldots, g) \in$ $\left.S_{n}^{d+1}\right\}$. An important special case of isotopism is the action of $\Delta_{n}^{d+1}$ on $\mathcal{H}_{n}^{d}$. This action is called isomorphism and its orbits are called isomorphism classes. If the hypercube is regarded as the table of values of a $d$-ary quasigroup on $[n]$, then isomorphisms of the hypercube correspond to standard isomorphisms of the quasigroup.

A further group action on a hypercube is provided by permutation of the elements of tuples. In this action, a permutation $\tau \in S_{d+1}$ maps the tuple $\left\langle v_{1}, v_{2}, \ldots, v_{d+1}\right\rangle$ onto the tuple $\left\langle v_{1}, v_{2}, \ldots, v_{d+1}\right\rangle^{\tau}=\left\langle w_{1}, w_{2}, \ldots, w_{d+1}\right\rangle$ where $w_{i^{\tau}}=v_{i}$ for $1 \leq i \leq d+1$. Here, and 
elsewhere, we use the superscript notation for the image of an object under a function; thus $i^{\tau}$ means $\tau(i)$, and $L^{\tau}$ is the image of $L$ obtained by applying $\tau$ to each of its tuples. Such images are the conjugates (also called parastrophes) of $L$.

An arbitrary combination of a conjugacy and an isotopism is called a paratopism (or paratopy). The set of all paratopisms corresponds to the wreath product $S_{n} 2 S_{d+1}$ in its natural action on $[n]^{d+1}$. The orbits of its action on the set of all hypercubes are called paratopy classes, main classes or species.

The stabilisers of a latin hypercube $L$ under isotopism, paratopism and isomorphism are known respectively as the autotopism group, autoparatopism group and automorphism group of $L$. We use respectively $\operatorname{Is}(L), \operatorname{Par}(L)$ and $\operatorname{Aut}(L)$ to denote these groups. For example, $\operatorname{Aut}(L)=\left\{\sigma \in \Delta_{n}^{d+1} \mid L^{\sigma}=L\right\}$.

One of the main objectives of this paper is enumeration of latin hypercubes and associated structures (such as $d$-ary quasigroups). The enumeration of latin squares has a lengthy and well known history for which we refer to the recent survey given in [18]. Considerably less work has been done for higher dimensions.

To count latin hypercubes it suffices to count reduced latin hypercubes and then apply the formula

$$
\left|\mathcal{H}_{n}^{d}\right|=n !(n-1) !^{d-1}\left|\mathcal{R}_{n}^{d}\right|
$$

One of the first works was by Gupta [9] who enumerated latin cubes by hand and obtained the incorrect value $\left|\mathcal{R}_{4}^{3}\right|=58$. The correct value $\left|\mathcal{R}_{4}^{3}\right|=64$ was subsequently obtained by Mullen and Weber [22] and much later by Jia and Qin [13], with both pairs of researchers attempting to count and classify latin cubes of orders 1 up to 5 .

Mullen and Weber [22] reported the numbers of reduced latin cubes of orders 1 up to 5 to be 1,1,1,64, 40246 and the numbers of isomorphism classes to be $1,1,1,19,1860$. They did not establish the number of isotopism classes for order 5 , but found the number for orders 1 to 4 respectively to be 1,1,1,12. Zinoviev and Zinoviev [28] found that there are 5 paratopy classes of latin cubes of order 4 . Our computations confirm the above numbers.

Apparently unaware of Mullen and Weber's work two decades earlier, Jia and Qin [13] attempted similar computations. They reported the same numbers of reduced latin cubes but gave the numbers of isotopism classes to be $1,1,1,15,479$. These last two values are incorrect.

A patent application by Ito [12] included values of $\left|\mathcal{R}_{4}^{d}\right|$ for $d \leq 5$ that agree with our computations (see Table 1), and also with the values found by Potapov and Krotov [23]. Potapov and Krotov also proved that

$$
3^{d+1} 2^{2^{d}+1} \leq\left|\mathcal{H}_{4}^{d}\right| \leq\left(3^{d+1}+1\right) 2^{2^{d}+1}
$$

for $d \geq 5$. 


\section{Counting equivalence classes}

The numbers of isotopy and paratopy classes of latin hypercubes are related to the total number of latin hypercubes via the sizes of the autotopism and autoparatopism groups, as in the following lemma whose proof is elementary.

Lemma 1. For any $L \in \mathcal{H}_{n}^{d}$, the paratopy class of $L$ contains

(a) $\frac{(d+1) !}{|\operatorname{Par}(L)| /|\operatorname{Is}(L)|}$ isotopy classes,

(b) $\frac{(d+1) ! n ! n^{d-1}}{|\operatorname{Par}(L)|}$ reduced latin hypercubes, and

(c) $\frac{(d+1) !(n !)^{d+1}}{|\operatorname{Par}(L)|}$ latin hypercubes.

In the case of isomorphism classes, the natural setting is that of quasigroups and loops. The procedure for counting isomorphism classes of ordinary quasigroups given in [18, Theorem 4] applies equally well to the $d$-ary case, and the same proof applies with obvious adaptions, so we state our first theorem without proof.

Let $\mathcal{I}_{n}^{d}$ be a set containing one hypercube from each isotopy class of $\mathcal{H}_{n}^{d}$, and let $\mathcal{M}_{n}^{d}$ be a set containing one hypercube from each paratopy class of $\mathcal{H}_{n}^{d}$.

Define the cycle structure of a permutation $\gamma$ to be the sequence $\left(n_{1}, n_{2}, \ldots\right)$, where $n_{i}$ is the number of cycles of length $i$ in $\gamma$. If $\sigma=\left(\sigma_{1}, \sigma_{2}, \ldots, \sigma_{d+1}\right)$ is a $(d+1)$-tuple of permutations, define $\psi(\sigma)$ as follows:

(i) If $\sigma_{1}, \sigma_{2}, \ldots, \sigma_{d+1}$ have the same cycle structure $\left(n_{1}, n_{2}, \ldots\right)$, then $\psi(\sigma)=\prod_{i} n_{i} ! i^{n_{i}}$;

(ii) otherwise, $\psi(\sigma)=0$.

Theorem 1. The number of isomorphism classes of d-ary quasigroups of order $n$ is

$$
\sum_{L \in \mathcal{I}_{n}^{d}} \frac{1}{|\operatorname{Is}(L)|} \sum_{\sigma \in \operatorname{Is}(L)} \psi(\sigma)^{d}=\sum_{L \in \mathcal{M}_{n}^{d}} \frac{(d+1) !}{|\operatorname{Par}(L)|} \sum_{\sigma \in \operatorname{Is}(L)} \psi(\sigma)^{d}
$$

As pointed out by a referee, some of these results are true in a more general setting. For some set $X$, let $\mathcal{F}_{n}^{d}(X)$ be the class of all functions from $[n]^{d+1}$ to $X$. A latin hypercube is such a function for $X=\{0,1\}$, namely the characteristic function of the set of $(d+1)$-tuples it comprises. Isotopy, paratopy and isomorphism are defined for $\mathcal{F}_{n}^{d}(X)$ by permutations of the domain $[n]^{d+1}$ in the same way as for latin hypercubes. We then have, with essentially the same proofs, that Lemma 1(a), (c) and Theorem 1 are true for any subclass of $\mathcal{F}_{n}^{d}(X)$ which is closed under paratopy.

While counting $d$-ary quasigroups required no new theory, it turns out that counting $d$-ary loops is less straightforward. The "obvious" extension of [18, Theorem 5] to the 
$d$-ary case does not work, primarily due to the possibility that loops have more than one identity element (see Section 5 for examples). Therefore, we need to develop a more complicated approach.

We say that two cells $\vec{u}$ and $\vec{v}$ of $L \in \mathcal{H}_{n}^{d}$ are line-equivalent if $\rho_{j}(\vec{u}, L)=\rho_{j}(\vec{v}, L)$ for all $j \in[d]$. If $x, y$ are both identity elements of a $d$-ary loop then $(x, x, \ldots, x)$ is line equivalent to $(y, y, \ldots, y)$.

For $1 \leq i \leq d+1$, define $L^{[i]}$ to be the conjugate of $L$ obtained by exchanging the $i$-th and $(d+1)$-th elements of tuples. This includes the case $L^{[d+1]}=L$.

Theorem 2. For $L \in \mathcal{H}_{n}^{d}$, choose $C(L)$ to be a set of cells that includes exactly one cell from each line-equivalence class of cells of $L$. For each cell $\vec{v}$, define

$$
N(\vec{v}, L)=\left|\left\{h \in \operatorname{Is}(L) \mid h^{\left(\rho_{1}, \rho_{2}, \ldots, \rho_{d}, 1\right)} \in \Delta_{n}^{d+1}\right\}\right|,
$$

where $\rho_{j}=\rho_{j}(\vec{v}, L)$ for each $j$, and 1 is the identity of $S_{n}$.

Then the number of isomorphism classes of d-ary loops of order $n$ is

$$
\sum_{L \in \mathcal{I}_{n}^{d}} \frac{1}{|\operatorname{Is}(L)|} \sum_{\vec{v} \in C(L)} N(\vec{v} ; L)=\sum_{L \in \mathcal{M}_{n}^{d}} \frac{d !}{|\operatorname{Par}(L)|} \sum_{t=1}^{d+1} \sum_{\vec{v} \in C\left(L^{[t]}\right)} N\left(\vec{v} ; L^{[t]}\right) .
$$

Proof. Let $\mathcal{L}_{n}^{d}$ consist of all the loops in $\mathcal{H}_{n}^{d}$. Note that we do not require any particular elements to be the identity elements. The number we seek is the number of equivalence classes of $\mathcal{L}_{n}^{d}$ under $\Delta_{n}^{d+1}$. By the Burnside-Frobenius Lemma, this is equal to the average number of loops fixed by an element of $\Delta_{n}^{d+1}$. Our task is therefore to find the number of pairs $(M, g)$ for $M \in \mathcal{L}_{n}^{d}$ and $g \in \Delta_{n}^{d+1}$, such that $M^{g}=M$.

We start by seeking the number of such pairs where $M$ is in the isotopy class of some fixed $L \in \mathcal{H}_{n}^{d}$. This isotopy class consists of all $L^{\sigma}$ for $\sigma \in S_{n}^{d+1}$, with the caveat that each hypercube appears for exactly $|\operatorname{Is}(L)|$ values of $\sigma$. Leaving that factor for later, we need to find the number of pairs $(\sigma, g)$ for $\sigma \in S_{n}^{d+1}$ and $g \in \Delta_{n}^{d+1}$, such that $L^{\sigma}$ is a loop and $L^{\sigma g}=L^{\sigma}$. The last condition is equivalent to $\sigma g \sigma^{-1} \in \operatorname{Is}(L)$.

Writing $h$ for $\sigma g \sigma^{-1}$, our problem reduces to counting all pairs $(\sigma, h)$ for $\sigma \in S_{n}^{d+1}$ and $h \in \operatorname{Is}(L)$ such that

(a) $L^{\sigma}$ is a loop, and

(b) $h^{\sigma} \in \Delta_{n}^{d+1}$.

We proceed by parameterizing those $\sigma$ satisfying (a). This is analogous to reducing a latin square, except that we do not require an identity element in the first position, and, indeed, we must remember that there can be more than one identity element.

- Choose a cell $\vec{v}$ and a permutation $\delta \in S_{n}$.

- Permute rows, columns, etc., so that the lines through cell $\vec{v}$ are all the identity permutation. 
- Apply $\delta \in S_{n}$ to each of the $d+1$ components.

The resulting loop has $L(\vec{v})^{\delta}$ as an identity element. Symbolically, we have

$$
\sigma=\sigma(\vec{v}, \delta)=\left(\rho_{1}(\vec{v}, L) \delta, \rho_{2}(\vec{v}, L) \delta, \ldots, \rho_{d}(\vec{v}, L) \delta, \delta\right) .
$$

It is easy to see that every $\sigma$ satisfying (a) has this form. However, this parameterisation has redundancies: $\sigma\left(\vec{u}, \delta^{\prime}\right)=\sigma(\vec{v}, \delta)$ if and only if $\vec{u}$ is line-equivalent to $\vec{v}$ and $\delta^{\prime}=\delta$.

Finally, for each $\sigma=\sigma(\vec{v}, \delta)$, we need to know how many $h \in \operatorname{Is}(L)$ satisfy (b). This does not depend on $\delta$ since $h^{\sigma(\vec{v}, \delta)} \in \Delta_{n}^{d+1} \Leftrightarrow h^{\sigma(\vec{v}, 1)} \in \Delta_{n}^{d+1}$.

We can now derive the first expression in (2). For each $L \in \mathcal{I}_{n}^{d}$, the number of pairs $(\sigma, h)$ satisfying (a) and (b) can be found by testing, for one $\vec{v}$ from each line-equivalence class of cell, whether $\sigma(\vec{v}, 1)$ satisfies (b). For each of those that pass the test, we have $n$ ! choices of $\delta$. Then we divide by $|\operatorname{Is}(L)|$ to account for the number of times each hypercube appears as $L^{\sigma}$ and by $\left|\Delta_{n}^{d+1}\right|=n$ ! as required by the Burnside-Frobenius Lemma.

The summand of the outside summation in the first half of (2) is not invariant under conjugacy, so converting to a sum over paratopy classes is not just a matter of applying some factors. However, the summand is invariant under conjugacies that leave the $(d+1)$-th position fixed. Therefore, we get all the information we need from the conjugates $L^{[1]}, L^{[2]}, \ldots, L^{[d+1]}$. Each of these represents $d !|\operatorname{Is}(L)| /|\operatorname{Par}(L)|$ isotopy classes, so the second half of $(2)$ is obtained.

In Table 1 we give the results of our enumeration of latin hypercubes, classified according to various notions of equivalence. A more detailed count of hypercubes, classified according to group sizes, is presented in the appendix. A representative of each of the paratopy classes covered by Table 1 can be found in [21]. In Table 2 we give the results of our enumeration of loops (up to isomorphism) according to the number of identity elements they have. In Section 5 we consider further the question of how many identity elements a loop can have. To save space, we have omitted a number of known values for $d=2$ and $n \in\{9,10,11\}$, see $[18,20]$.

\section{Construction method}

To reduce the probability of error, all the computations were carried out independently by the two authors using slightly different algorithms. We now describe the first algorithm used.

Let $L \in \mathcal{H}_{n}^{d}$. For $1 \leq k \leq n$, the $k$-th hyperplane of $L$ is $L^{(k)} \in \mathcal{H}_{n}^{d-1}$ defined by $L^{(k)}\left(i_{1}, \ldots, i_{d-1}\right)=L\left(i_{1}, \ldots, i_{d-1}, k\right)$ for all $i_{1}, \ldots, i_{d-1}$.

Next we define an ordering on latin hypercubes. The hypercube $L$ can be specified by 


\begin{tabular}{cc|cccc}
$d$ & $n$ & reduced & isotopy classes & paratopy classes & quasigroups \\
\hline 2 & 2 & 1 & 1 & 1 & 1 \\
2 & 3 & 1 & 1 & 1 & 5 \\
2 & 4 & 4 & 2 & 2 & 35 \\
2 & 5 & 56 & 2 & 2 & 1411 \\
2 & 6 & 9408 & 22 & 12 & 1130531 \\
2 & 7 & 16942080 & 564 & 147 & 12198455835 \\
2 & 8 & 535281401856 & 1676267 & 283657 & 2697818331680661 \\
\hline 3 & 2 & 1 & 1 & 1 & 1 \\
3 & 3 & 1 & 1 & 1 & 11 \\
3 & 4 & 64 & 12 & 5 & 2589 \\
3 & 5 & 40246 & 59 & 15 & 23192922 \\
3 & 6 & 95909896152 & 5678334 & 264248 & 1381105636226980 \\
\hline 4 & 2 & 1 & 1 & 1 & 1 \\
4 & 3 & 1 & 1 & 1 & 1565243 \\
4 & 4 & 7132 & 328 & 26 & 1 \\
4 & 5 & 31503556 & 5466 & 86 & 43 \\
\hline 5 & 2 & 1 & 1 & 1 & 263347981121 \\
5 & 3 & 1 & 1 & 1 & 16751644838639300 \\
5 & 4 & 201538000 & 2133586 & 4785 & 1 \\
5 & 5 & 50490811256 & 1501786 & 3102 & 1 \\
\hline 6 & 2 & 1 & 1 & 1 & \\
6 & 3 & 1 & 1 & & 135509352937 \\
\hline
\end{tabular}

Table 1: Number of reduced latin hypercubes, isotopy classes of latin hypercubes, paratopy classes of latin hypercubes and isomorphism classes of quasigroups for small orders $n$ and dimensions $d$.

listing the symbols in each of the $n^{d}$ cells in a particular order:

$$
\begin{aligned}
\Sigma(L)=[ & L(1,1,1, \ldots, 1), L(2,1,1, \ldots, 1), \ldots, L(n, 1,1, \ldots, 1), \\
& L(1,2,1, \ldots, 1), L(2,2,1, \ldots, 1), \ldots, L(n, 2,1, \ldots, 1), \\
& L(1,3,1, \ldots, 1), \ldots \\
& L(1, n, 1, \ldots, 1), L(2, n, 1, \ldots, 1), \ldots, L(n, n, 1, \ldots, 1), \\
& L(1,1,2, \ldots, 1), \ldots \\
& L(1, n, n, \ldots, n), L(2, n, n, \ldots, n), \ldots, L(n, n, n, \ldots, n)] .
\end{aligned}
$$

The order of the cells (earlier indices varying faster) is important for what follows. We call $L$ isotopy-minimal if $\Sigma(L) \leq \Sigma\left(L^{\prime}\right)$ for every $L^{\prime}$ in the isotopy class of $L$. Similarly, $L$ is paratopy-minimal if $\Sigma(L) \leq \Sigma\left(L^{\prime}\right)$ for every $L^{\prime}$ in the paratopy class of $L$. In each case, 


\begin{tabular}{|c|c|c|c|c|c|c|c|c|}
\hline \multirow[b]{2}{*}{$d$} & \multirow[b]{2}{*}{$n$} & \multicolumn{6}{|c|}{ Number of identity elements } & \multirow[b]{2}{*}{ Total } \\
\hline & & 1 & 2 & 3 & 4 & 5 & 6 & \\
\hline 2 & 2 & 1 & & & & & & 1 \\
\hline 2 & 3 & 1 & & & & & & 1 \\
\hline 2 & 4 & 2 & & & & & & 2 \\
\hline 2 & 5 & 6 & & & & & & 6 \\
\hline 2 & 6 & 109 & & & & & & 109 \\
\hline 2 & 7 & 23746 & & & & & & 23746 \\
\hline 2 & 8 & 106228849 & & & & & & 106228849 \\
\hline 2 & 9 & 9365022303540 & & & & & & 9365022303540 \\
\hline 3 & 2 & 0 & 1 & & & & & 1 \\
\hline 3 & 3 & 1 & 0 & 0 & & & & 1 \\
\hline 3 & 4 & 3 & 15 & 0 & 1 & & & 19 \\
\hline 3 & 5 & 1826 & 32 & 1 & 0 & 1 & & 1860 \\
\hline 3 & 6 & 797971315 & 1422290 & 525 & 90 & 0 & 6 & 799394226 \\
\hline 4 & 2 & 1 & 0 & & & & & 1 \\
\hline 4 & 3 & 0 & 0 & 1 & & & & 1 \\
\hline 4 & 4 & 2213 & 0 & 0 & 0 & & & 2213 \\
\hline 4 & 5 & 1349704 & 9 & 0 & 0 & 0 & & 1349713 \\
\hline 5 & 2 & 0 & 1 & & & & & 1 \\
\hline 5 & 3 & 1 & 0 & 0 & & & & 1 \\
\hline 5 & 4 & 34140 & 66060918 & 0 & 524800 & & & 66619858 \\
\hline 5 & 5 & 2122915806 & 805040 & 280 & 0 & 70 & & 2123721196 \\
\hline 6 & 2 & 1 & 0 & & & & & 1 \\
\hline 6 & 3 & 1 & 0 & 0 & & & & 1 \\
\hline
\end{tabular}

Table 2: Counts of loops according to their number of identity elements

the comparison $\leq$ is lexicographic order. The following properties follow easily from the definitions.

Lemma 2. Let $L$ be a latin hypercube. Then the following hold.

(i) There is exactly one isotopy-minimal hypercube in each isotopy class and exactly one paratopy-minimal hypercube in each paratopy class.

(ii) If $L$ is paratopy-minimal, then $L$ is isotopy-minimal.

(iii) If $L$ is isotopy-minimal, then $L$ is reduced.

(iv) If $L$ is paratopy-minimal in $\mathcal{H}_{n}^{d}$, then $L^{(1)}$ is paratopy-minimal in $\mathcal{H}_{n}^{d-1}$.

The aim of the computation was to find all paratopy-minimal hypercubes $L \in \mathcal{H}_{n}^{d}$. As permitted by Lemma 2(iv), we took the paratopy-minimal hypercubes in $\mathcal{H}_{n}^{d-1}$ as $L^{(1)}$. 
Then we extended $L^{(1)}$ to $L$ in all possible ways such that $L$ was reduced. Finally, we rejected $L$ if it was not paratopy-minimal.

To check that $L$ is paratopy-minimal, we verified that $\Sigma(L) \leq \Sigma\left(L^{\gamma}\right)$ for each of the $n^{d-1}(d+1) ! n$ ! paratopisms $\gamma$ that take $L$ to a reduced hypercube. These paratopisms have the form $\gamma=\gamma(\vec{v}, \delta, L)=\sigma(\vec{v}, \delta) \tau$, where $\tau$ is one of the $(d+1)$ ! conjugacies, and $\sigma(\vec{v}, \delta)$ is defined as in (3) with $\delta \in S_{n}$ such that $L(\vec{v})^{\delta}=1$. In this case, the redundancy issue noted just after (3) does not occur: all these $n^{d-1}(d+1) ! n$ ! paratopisms are different. To see this, note that the condition $L(\vec{v})^{\delta}=1$ implies that $\sigma(\vec{v}, \delta)$ maps position $\vec{v}$ onto position $(1,1, \ldots, 1)$. If $\gamma=\gamma(\vec{v}, \delta, L)$ was found such that $\Sigma\left(L^{\gamma}\right)<\Sigma(L)$, thereby rejecting $L$ as not paratopy-minimal, the next candidate hypercube $L^{\prime}$ was first tested with $\gamma\left(\vec{v}, \delta, L^{\prime}\right)$. This often rejected $L^{\prime}$ as well, giving a useful speedup.

If the minimality test for $L$ was passed, we had also found the autoparatopism group: $\operatorname{Par}(L)$ consists of those $\gamma$ for which $\Sigma\left(L^{\gamma}\right)=\Sigma(L)$.

The second algorithm used to count $\left|\mathcal{H}_{n}^{d}\right|$ was similar to the first in that it generated a catalogue of paratopy class representatives by extending all paratopy class representatives from $\mathcal{H}_{n}^{d-1}$ in all ways that produced a reduced hypercube. However, the isomorphism testing was different. The reduced hypercubes were canonically labelled using nauty [19] in much the same way as latin squares were treated in [18]. Any hypercube was rejected if its canonical labelling was identical to that of a previously constructed hypercube. The autoparatopism group of each hypercube was calculated by nauty while finding the canonical labelling.

It is clear that both these generation methods are quite crude, but the use of more sophisticated techniques, such as applying a minimality test after each hyperplane is added, would not help very much with these small values of $n$ and $d$. In particular, we do not think they would improve the efficiency enough to make hypercubes with higher order and/or dimension enumerable with present computing power.

\section{$4 \quad$ Number of quasigroups of order 3}

In this section we count the $d$-ary quasigroups of order $n=3$, for arbitrary $d$. Before developing that result we need to introduce a notion of linearity.

We say that $H \in \mathcal{H}_{n}^{d}$ is linear if there exist $s \in[n]$ and $c_{i} \in[n]$ for $i \in[d]$, such that

$$
H\left(x_{1}, x_{2}, \ldots, x_{d}\right) \equiv s+\sum_{i=1}^{d} c_{i} x_{i} \quad(\bmod n),
$$

for each $\left(x_{1}, x_{2}, \ldots, x_{d}\right) \in[n]^{d}$. We say that a quasigroup/loop is linear if its corresponding hypercube is linear. For (4) to define a latin hypercube it is necessary and sufficient that each $c_{i}$ should be relatively prime to $n$. Let $\phi$ denote Euler's phi function. It follows that 
there are exactly $n(\phi(n))^{d}$ linear hypercubes in $\mathcal{H}_{n}^{d}$, since we have $n$ choices for $s$ and $\phi(n)$ choices for each $c_{i}$.

For $n \leq 3$ and arbitrary $d$ it is easy to prove by induction on $d$ that every hypercube in $\mathcal{H}_{n}^{d}$ is linear. This conclusion also follows from the values of $\left|\mathcal{H}_{n}^{d}\right|$ as given, for example, by Finizio and Lewis [7] or Laywine and Mullen [16, p.224].

In our next theorem we will use the fact that each of the $2^{d} 3$ hypercubes in $\mathcal{H}_{3}^{d}$ is linear to count the $d$-ary quasigroups of order 3 . Our result is phrased in terms of the Jacobsthal sequence, which is defined by $a_{n}=\frac{1}{3}\left(2^{n}+(-1)^{n+1}\right)$, or alternatively by the recurrence

$$
a_{n}=a_{n-1}+2 a_{n-2} \quad \text { with } \quad a_{1}=a_{2}=1 .
$$

This is sequence A001045 in Sloane's On-Line Encyclopedia of Integer Sequences [25], which lists many combinatorial objects counted by the sequence. Here we extend that list.

Theorem 3. Let $\mathcal{Q}_{3, g}^{d}$ be the set of d-ary quasigroups of order 3 with an automorphism group of orderg. Then $\left|\mathcal{Q}_{3,2}^{d}\right|=a_{d+1},\left|\mathcal{Q}_{3,3}^{d}\right|=\left|\mathcal{Q}_{3,6}^{d}\right|=a_{d}$ and $\left|\mathcal{Q}_{3, g}^{d}\right|=0$ for $g \notin\{2,3,6\}$. Hence $\left|\mathcal{Q}_{3}^{d}\right|=a_{d+2}$.

Proof. First note that the result for $\left|\mathcal{Q}_{3}^{d}\right|$ follows from the other results and (5), since $\left|\mathcal{Q}_{3}^{d}\right|=\sum_{g}\left|\mathcal{Q}_{3, g}^{d}\right|$.

Let $\mathcal{H}_{3, g}^{d}$ be the set of $d$-dimensional hypercubes of order $n$ for which the corresponding quasigroup is in $\mathcal{Q}_{3, g}^{d}$. Clearly, $\left|\mathcal{Q}_{3, g}^{d}\right|=\left|\mathcal{H}_{3, g}^{d}\right| g / 3$ ! .

Consider the diagonal cells of an arbitrary $H \in \mathcal{H}_{3}^{d}$. Specifically, for $i \in\{1,2,3\}$ define $u_{i}$ by $\left\langle i, i, i, \ldots, i, u_{i}\right\rangle \in H$. Since $H$ must be linear, we find that $u_{2}-u_{1} \equiv u_{3}-u_{2} \equiv u_{1}-u_{3}$ $(\bmod 3)$.

We now argue that $u_{k}=k$ if and only if $H$ possesses an automorphism $\tau$ fixing $k$ and swapping the other two elements of $\{1,2,3\}$.

Certainly if $H$ has such an automorphism then it maps $\left\langle k, k, \ldots, k, u_{k}\right\rangle$ to $\langle k, k, \ldots, k$, $\left.\tau\left(u_{k}\right)\right\rangle$, from which we infer that $u_{k}=k$, the only fixed point of $\tau$.

So assume that $u_{k}=k$ and define $\tau:[3] \rightarrow[3]$ by $\tau(x) \equiv-k-x(\bmod 3)$. Note that $\tau$ fixes $k$ and swaps the elements of $[3] \backslash\{k\}$. Since $H$ is linear we can assume the existence of $s, c_{i}$ satisfying (4). Given that $u_{k}=k$, this implies that $k=s+k \sum c_{i}$. Now if we apply the isomorphism $\tau$ to a general element $\left\langle x_{1}, x_{2}, \ldots, x_{d}, s+\sum c_{i} x_{i}\right\rangle$ of $H$, it maps that element to

$$
\left\langle-k-x_{1},-k-x_{2}, \ldots,-k-x_{d},-k-s-\sum c_{i} x_{i}\right\rangle
$$

where all coordinates should be calculated mod 3. However, this is an element of $H$ since $s+\sum c_{i}\left(-k-x_{i}\right)=s-k \sum c_{i}-\sum c_{i} x_{i}=s+(s-k)-\sum c_{i} x_{i} \equiv-k-s-\sum c_{i} x_{i}$ 
mod 3. We conclude that $\tau$ is indeed an automorphism, as we contended.

At this point we separate into three cases. Define $\alpha \in[3]$ by $\alpha \equiv u_{2}-u_{1} \equiv u_{3}-u_{2} \equiv$ $u_{1}-u_{3}(\bmod 3)$.

Case 1: $\quad \alpha \neq 1$

By the definition of $\alpha$ there exists a unique $k \in\{1,2,3\}$ such that $u_{k}=k$. This means $\operatorname{Aut}(H)$ contains a unique transposition and hence $|\operatorname{Aut}(H)|=2$.

Case 2: $\quad \alpha=1$ and there exists $k \in\{1,2,3\}$ such that $u_{k}=k$.

Since $\alpha=1$ we know $u_{k}=k$ for all $k \in\{1,2,3\}$. This means $\operatorname{Aut}(H)$ contains three transpositions and hence $|\operatorname{Aut}(H)|=6$.

Case 3: $\quad \alpha=1$ and there does not exist $k \in\{1,2,3\}$ such that $u_{k}=k$.

In this case $\operatorname{Aut}(H)$ contains no transpositions. However, by applying the permutation $\pi: u_{k} \rightarrow k$ to the symbols of $H$ we obtain a hypercube $H^{\prime}$ to which case 2 applies. That means that $\pi$ is an automorphism of $H^{\prime}$, and hence also of $H$. We deduce that $|\operatorname{Aut}(H)|=3$.

Moreover there must be exactly twice as many hypercubes in case 3 as there are in case 2. Any example from case 2 can be turned into an example from case 3 by applying either (123) or (321) to its symbols. Exactly one of these permutations will map an example from case 3 to one from case 2 . It follows that $\left|\mathcal{H}_{3,3}^{d}\right|=2\left|\mathcal{H}_{3,6}^{d}\right|$ and thus $\left|\mathcal{Q}_{3,3}^{d}\right|=\left|\mathcal{Q}_{3,6}^{d}\right|$.

We now consider how a $(d-1)$-dimensional hypercube $H$ can be embedded as the first hyperplane of a $d$-dimensional hypercube $H^{\prime}$. Since our hypercubes are linear there is only one choice to make, namely whether $c_{d}=1$ or $c_{d}=2$. If $H$ is in case 2 or 3 then for either choice $H^{\prime}$ must fall into case 1 . On the other hand, if $H$ is in case 1 then there is exactly one choice of $c_{d}$ so that $H^{\prime}$ is also in case 1 . Compiling this information we get

$$
\begin{aligned}
\left|\mathcal{H}_{3,2}^{d}\right| & =2\left|\mathcal{H}_{3,3}^{d-1} \cup \mathcal{H}_{3,6}^{d-1}\right|+\left|\mathcal{H}_{3,2}^{d-1}\right| \\
\left|\mathcal{H}_{3,3}^{d} \cup \mathcal{H}_{3,6}^{d}\right| & =\left|\mathcal{H}_{3,2}^{d-1}\right|
\end{aligned}
$$

and hence

$$
\begin{aligned}
\left|\mathcal{Q}_{3,2}^{d}\right| & =2\left|\mathcal{Q}_{3,3}^{d-1}\right|+\left|\mathcal{Q}_{3,2}^{d-1}\right| \\
\left|\mathcal{Q}_{3,3}^{d}\right| & =\left|\mathcal{Q}_{3,2}^{d-1}\right| .
\end{aligned}
$$

The theorem now follows from (5) by induction on $d$.

\section{$5 \quad$ Number of identity elements}

It is well known that a binary quasigroup can have at most one identity element but that $d$-ary quasigroups for $d>2$ can have multiple identity elements. In this section we 
investigate constructions which produce quasigroups with a specific number of identity elements.

It is beyond the scope of this paper to provide a complete answer to the question of for which $n, d$ and $i$ there exists a quasigroup in $\mathcal{Q}_{n}^{d}$ with exactly $i$ identity elements. We set the more modest goal of, at a minimum, constructing at least one quasigroup in all cases for which Table 2 claims existence. Our methods and results represent a step toward an eventual solution of the more general question. We begin with some notation that will be used throughout this section.

Suppose $A \in \mathcal{Q}_{n}^{\alpha}$ and $B \in \mathcal{Q}_{n}^{\beta}$. We define a new quasigroup in $\mathcal{Q}_{n}^{\alpha+\beta-1}$, which we call the composition of $A$ and $B$ and write as $A \vdash B$, by

$$
(A \vdash B)\left(x_{1}, \ldots, x_{\alpha+\beta-1}\right)=A\left(B\left(x_{1}, \ldots, x_{\beta}\right), x_{\beta+1}, \ldots, x_{\alpha+\beta-1}\right) .
$$

Also, for any quasigroup $A$ and positive integer $c$ we define $A^{c}$ inductively by $A^{1}=A$ and $A^{c}=A^{c-1} \vdash A$ for $c>1$. In particular, if $\mathbb{Z}_{n}$ denotes the quasigroup in $\mathcal{Q}_{n}^{2}$ defined by $\mathbb{Z}_{n}(x, y) \equiv x+y(\bmod n)$, then $\left(\mathbb{Z}_{n}\right)^{c}$ is the quasigroup in $\mathcal{Q}_{n}^{c+1}$ which is evaluated simply by adding coordinates modulo $n$.

The next lemma makes some observations about the number of identity elements in compositions. The (entirely elementary) proof will be omitted. In part 3, the meaning of total loop is a loop in which every element is an identity element.

\section{Lemma 3.}

1. $\left(\mathbb{Z}_{n}\right)^{c} \in \mathcal{Q}_{n}^{c+1}$ has $\operatorname{gcd}(n, c)$ identity elements.

2. If $x$ is an identity element in both $A$ and $B$ then $x$ is an identity element in $A \vdash B$. The converse is false, as can easily be seen by considering observation 1.

3. Suppose $A$ is a total loop. Then $x$ is an identity element in $A \vdash B$ if and only if $x$ is an identity element in $B$.

The following result proves, among other things, existence of total loops in many cases.

Theorem 4. Suppose that $n, d, i$ are positive integers satisfying

- $n \notin\{3,7\}$,

- $d \geq 3$ and $d$ is odd,

- $i \equiv n(\bmod 2)$ or $i=2$.

Then there exists a loop in $\mathcal{Q}_{n}^{d}$ with exactly $i$ identity elements. 
Proof. For any odd $d>1$ and any $n \notin\{3,7\}$ Teirlinck [26] showed the existence in $\mathcal{Q}_{n}^{d}$ of a 2-idempotent quasigroup, namely a quasigroup in which each subset of cardinality 1 or 2 is a subquasigroup. It is easy to see that every 2-idempotent quasigroup is a total loop, proving the case $i=n$.

Suppose $i \equiv n(\bmod 2)$. In a 2-idempotent quasigroup, we can replace each of the subquasigroups on the pairs $\{i+1, i+2\},\{i+3, i+4\}, \ldots,\{n-1, n\}$ by the other possible subquasigroup on the same two elements. Doing so results in a new quasigroup in which none of $i+1, \ldots, n$ is an identity element. However, no tuple containing any element less than $i+1$ has been altered, so the elements $1, \ldots, i$ are all still identity elements.

It remains to show the $i=2$ case. Again we start with a 2-idempotent quasigroup. Suppose after replacing the subquasigroup on the elements $\{1,2\}$ by the other possible subquasigroup on those elements, we arrive at a quasigroup $Q$. Now define $Q^{\prime}$ by

$$
Q^{\prime}(\vec{x})= \begin{cases}1 & \text { if } Q(\vec{x})=2, \\ 2 & \text { if } Q(\vec{x})=1 \\ Q(\vec{x}) & \text { otherwise }\end{cases}
$$

Then $Q^{\prime}$ will be a quasigroup in which 1 and 2 are identity elements. However, if $x \notin\{1,2\}$ then $Q^{\prime}(1, x, x, \ldots, x)=2$ so $x$ is not an identity element.

Teirlinck left open the case of existence when $n=7$, but it is easy to check by computer that no total loop (and hence no 2-idempotent quasigroup) exists in $\mathcal{Q}_{7}^{3}$. Despite this, we will show that a total loop exists in $\mathcal{Q}_{7}^{d}$ for all sufficiently large $d$. Also, total loops (unlike 2-idempotent quasigroups) can exist in even dimensions. Consider $S \in \mathcal{Q}_{7}^{3}$ given by

$$
\begin{aligned}
S= & {[5671234.6237415 .7364152 .1743526 .2415763 .3152647 .4526371 .} \\
& 6237415.2345671 .3471526 .7514263 .4652137 .1726354 .5163742 . \\
& 7364152.3471526 .6712345 .4125637 .1536274 .5243761 .2657413 . \\
& 1743526.7514263 .4125637 .3456712 .5267341 .2631475 .6372154 . \\
& 2415763.4652137 .1536274 .5267341 .7123456 .6374512 .3741625 . \\
& 3152647.1726354 .5243761 .2631475 .6374512 .4567123 .7415236 . \\
& 4526371.5163742 .2657413 .6372154 .3741625 .7415236 .1234567] .
\end{aligned}
$$

It is easy to check that $\left(\mathbb{Z}_{7}\right)^{3} \vdash S$ is a total loop in $\mathcal{Q}_{7}^{6}$. By composing this total loop with itself we can then produce total loops in $\mathcal{Q}_{7}^{6+5 k}$ for any integer $k \geq 0$.

Theorem 5. For each odd $n>3$ there exists a $D$ such that $\mathcal{Q}_{n}^{d}$ contains a total loop for all $d \geq D$. 
Proof. We first show that for $n=7$ we can choose $D=25$. For arbitrary positive integers $k, c$ we have shown above the existence of a total loop $T \in \mathcal{Q}_{7}^{5 k+1}$ and also noted in Lemma 3 that $\left(\mathbb{Z}_{7}\right)^{7 c}$ is a total loop in $\mathcal{Q}_{7}^{7 c+1}$. Forming $T \vdash\left(\mathbb{Z}_{7}\right)^{7 c}$ then produces a total loop in $\mathcal{Q}_{7}^{7 c+5 k+1}$. Every integer exceeding 24 can be written in the form $7 c+5 k+1$ for non-negative integers $c, k$ (see, for example, Theorem 3.15.1 in [27]).

The argument for odd $n \notin\{3,7\}$ is similar, but we use a total loop $T \in \mathcal{Q}_{n}^{2 k+1}$ instead (whose existence is guaranteed by Theorem 4). Using $T \vdash\left(\mathbb{Z}_{n}\right)^{n c}$ gives a total loop in $\mathcal{Q}_{n}^{n c+2 k+1}$. Since $n$ is odd, every integer exceeding $n-1$ can be written in the form $n c+2 k+1$ for non-negative integers $c, k$. Thus we may take $D=n$.

Next we consider quasigroups in which there is (only) one element which is not an identity element. It seems plausible, given the data from Table 2, that the following is the only general restriction on how many identity elements there can be in a ternary quasigroup.

Lemma 4. A 3-ary quasigroup of order $n$ cannot have exactly $n-1$ identity elements.

Proof. Let $Q$ be a 3-ary quasigroup of order $n$ on the symbols $[n]$ in which every $x \in$ $[n] \backslash\{u\}$ is an identity element. Take an arbitrary $v \in[n] \backslash\{u\}$. Then there exists $x \in[n]$ such that $\langle v, x, u, v\rangle \in Q$. If $x \neq u$ this contradicts the fact that $\langle v, x, x, v\rangle \in Q$ since $x$ is an identity element. We conclude that $\langle v, u, u, v\rangle \in Q$, and a similar argument shows that $\langle u, v, u, v\rangle \in Q$ and $\langle u, u, v, v\rangle \in Q$. Finally, there is some $x \in[n]$ such that $\langle x, u, u, u\rangle \in Q$. If $x \neq u$ this contradicts $\langle x, u, u, x\rangle \in Q$, so we must have $\langle u, u, u, u\rangle \in Q$. We have shown that $u$ is an identity element, so if $Q$ has at least $n-1$ identity elements then it must have $n$ identity elements.

The data in Table 2 is consistent with a generalisation of Lemma 4 to some higher dimensions. However, no such generalisation is possible.

Lemma 5. For every $d>3$ there exists an $n$ and $a Q \in \mathcal{Q}_{n}^{d}$ such that $Q$ has exactly $n-1$ identity elements.

Proof. We first show that 5 -ary quasigroups can have exactly $n-1$ identity elements. Let

$$
\begin{aligned}
E=[ & 78123456.85274163 .12385674 .27816345 .34567812 .41638527 .56741238 .63452781 . \\
& 85274163.56781234 .27416385 .78123456 .41638527 .12345678 .63852741 .34567812 . \\
& 12385674.27416385 .34567812 .81634527 .56741238 .63852741 .78123456 .45278163 . \\
& 27816345.38527416 .81634527 .12345678 .63452781 .74163852 .45278163 .56781234 . \\
& 34567812.41638527 .56741238 .63452781 .78123456 .85274163 .12385674 .27816345 . \\
& 41638527.72143658 .63852741 .34567812 .85274163 .56781234 .27416385 .18325476 . \\
& 56741238.63852741 .78123456 .45278163 .12385674 .27416385 .34567812 .81634527 . \\
& 63452781.14365872 .45278163 .56781234 .27816345 .38527416 .81634527 .72143658]
\end{aligned}
$$


Now $Q \in \mathcal{Q}_{8}^{5}$ defined by $Q=\left(\mathbb{Z}_{8}\right)^{2} \vdash E$ has exactly 7 identity elements (the element 8 is not an identity element, since $Q(1,8,8,8,8)=7)$. By Theorem 4 , there is a total loop $T \in \mathcal{Q}_{8}^{2 k+1}$ for all $k \geq 1$. Then by Lemma $3, T \vdash Q$ is a loop in $\mathcal{Q}_{8}^{2 k+5}$ with exactly 7 identity elements. Thus we have the required example whenever the dimension is odd.

For any even $d,\left(\mathbb{Z}_{2}\right)^{d-1}$ provides a (rather trivial) example. By Lemma 3 , it is a $d$-ary quasigroup of order 2 with one identity element.

Our next result shows among other things that the $n=2$ examples used to prove Lemma 5 are the only linear examples of order $n$ quasigroups with $n-1$ identity elements.

Lemma 6. The number of identity elements in a linear d-ary loop of order $n$ divides $n$.

Proof. Suppose $L$ is a linear $d$-ary loop of order $n$, defined by (4). Since $L$ is a loop it possesses at least one identity element, say $\iota$. Then $\langle y, \iota, \iota, \ldots, \iota, y\rangle \in L$ for all $y \in[n]$ which implies that

$$
s+\iota \sum_{i=2}^{d} c_{i}+c_{1} y \equiv y \quad(\bmod n) .
$$

As (6) must hold for all $y$ we conclude that $c_{1}=1$. A similar argument shows that $c_{j}=1$ for all $j \in[d]$. Hence $(6)$ reduces to $s+(d-1) \iota \equiv 0(\bmod n)$. This recurrence either has no solution for $\iota$ (impossible since we know $L$ has an identity element), or its number of solutions is a divisor of $n$. It is obvious that each solution will yield a (different) identity element.

Having considered loops with a high number of identity elements, we turn briefly to the other end of the spectrum. It is a trivial matter for any $n \geq 3$ and $d \geq 2$ to create a linear quasigroup with no identity elements. For example, take $c_{i}=-1$ for all $i$ in (4), and consider the proof of Lemma 6 above. Building quasigroups with a unique identity element is also easy.

Theorem 6. For all $n \geq 4$ and $d \geq 2$ there exists a loop in $\mathcal{Q}_{n}^{d}$ with a unique identity element.

Proof. The result for $d=2$ is well known. For $d=3$ and odd $n$ we can use $\left(\mathbb{Z}_{n}\right)^{2}$, by Lemma 3.

Suppose $n$ is even and $n>2$. Let $Z_{n}^{\prime}=\mathbb{Z}_{n}^{(\tau, \tau, \tau)}$ be the binary quasigroup isomorphic to $\mathbb{Z}_{n}$ by applying the transposition $\tau=\left(1 \frac{n}{2}\right)$ uniformly to the triples. Now consider $Q=\mathbb{Z}_{n} \vdash Z_{n}^{\prime}$. The element $n$ is an identity element of $\mathbb{Z}_{n}, Z_{n}^{\prime}$ and hence also of $Q$, by Lemma 3 . For $x \notin\left\{\frac{n}{2}, n\right\}$, we have $Q(x, n, x) \equiv Z_{n}^{\prime}(x, n)+x \equiv 2 x \not \equiv n(\bmod n)$, so $x$ is not an identity element. Moreover, $\frac{n}{2}$ is not an identity element either, since $Q\left(\frac{n}{2}, \frac{n}{2}, n\right)=Z^{\prime}\left(\frac{n}{2}, \frac{n}{2}\right)=2 \neq n$.

We have thus shown the theorem for $d \leq 3$. From these base cases the theorem now follows for $d>3$ and $n \neq 7$ by composition with a total loop whose existence is guaranteed 
by Theorem 4 . For $n=7$, we use the base cases $\left(\mathbb{Z}_{7}\right)^{c}$ for $c \in[6]$, each of which has a unique identity element, by Lemma 3. By composing these with the total loops from $\mathcal{Q}_{7}^{5 k+1}$, we generate all the required examples for $n=7$.

We now describe examples of quasigroups with each of the possible number of identity elements shown in Table 2.

If $n \leq 3$ then part 1 of Lemma 3 provides the examples we need. So we can assume henceforth that $n \in\{4,5,6\}$. In particular this means there exists a total loop in $\mathcal{Q}_{n}^{3}$, by Theorem 4 , and a loop with a unique identity element in $\mathcal{Q}_{n}^{d}$ for all $d \geq 2$, by Theorem 6 . This provides the required examples for $d=4$, except that we need a quasigroup in $\mathcal{Q}_{5}^{4}$ with two identity elements. Let

$$
\begin{aligned}
Q_{a} & =[12345.23451 .34512 .45123 .51234] \\
Q_{b} & =[12345.25413 .34251 .41532 .53124] \\
Q_{c} & =[12345.25413 .34521 .43152 .51234]
\end{aligned}
$$

Then the quasigroup $Q\left(x_{1}, x_{2}, x_{3}, x_{4}\right)=Q_{c}\left(Q_{a}\left(x_{1}, x_{2}\right), Q_{b}\left(x_{3}, x_{4}\right)\right)$, has 2 identity elements, as required.

By composition with a total loop we will obtain all required examples for $d=5$ as soon as we find the examples for $d=3$. Moreover, most of the examples needed for $d=3$ can be found by applying Theorem 4 and Theorem 6 . Only one case remains, namely we need an example in $\mathcal{Q}_{6}^{3}$ with 3 identity elements. In fact, we give examples of all possible numbers of identity elements in $\mathcal{Q}_{6}^{3}$. Let

$$
\begin{aligned}
& Q_{0}=[124653.213546 .431265 .652134 .546312 .365421] \\
& Q_{1}=[123456.214365 .632514 .351642 .465231 .546123] \\
& Q_{2}=[123456.214365 .635214 .356142 .542631 .461523] \\
& Q_{3}=[123456.214365 .632541 .351624 .546132 .465213] \\
& Q_{4}=[123456.214365 .632514 .351642 .546231 .465123] \\
& Q_{6}=[123456.214365 .632541 .351624 .546213 .465132]
\end{aligned}
$$

For each $i \in\{0,1,2,3,4,6\}$, the quasigroup $Q_{i} \vdash Q_{0}$ has exactly $i$ identity elements.

\section{Incompletable latin cuboids}

A natural method of building latin hypercubes is to add hyperplanes one at a time, as we did in Section 3. We call the intermediate objects thereby created latin hypercuboids. In the three-dimensional case, Kochol [14] used the name "latin parallelepipeds". 
There is celebrated result due to Marshall Hall [10] that every latin rectangle is completable to a latin square. However, the equivalent statement is not true in higher dimensions. Kochol [14] proved that for any $k$ and $n$ satisfying $\frac{1}{2} n<k \leq n-2$ there is an $n \times n \times k$ latin cuboid which cannot be completed to an $n \times n \times n$ latin cube. Although he did not say so, it is simple to use such examples to create non-completable $n \times n \times \cdots \times n \times k$ latin hypercuboids in higher dimensions.

It is an open question how "thin" an non-completable latin hypercuboid can be. In acknowledging that his theorem is not best possible, Kochol [14] alluded to an example of an non-completable $5 \times 5 \times 2$ latin cuboid, although it seems this example might have never been published. Below we give examples of non-completable $5 \times 5 \times 2,6 \times 6 \times 2$, $7 \times 7 \times 3$ and $8 \times 8 \times 4$ latin cuboids. In the latter two cases it remains open whether there are thinner examples. The $5 \times 5 \times 2$ cubiod cannot be extended even to 3 layers, while the other examples can be extended to $n \times n \times(n-2)$ latin cuboids, but no further.

[12345.21453.34521.45132.53214.

21453.13542.52134.34215.45321]

[123456.214365.345612.436521.561234.652143.

214365.125643.456231.362154.643512.531426]

[1632745.6173452.2356174.5264317.3417526.7541263.4725631.

2147653.7456231.3715462.6523174.4362715.1634527.5271346.

3715426.1624573.6243751.4176235.2531647.5467312.7352164]

[14257638.68421375.26875413.57368241.85643127.31784562.43512786.72136854.

26813457.47286531 .38564172 .71635824 .13758246 .62341785 .54127368 .85472613$.

31462785.14738256.62147538.83251467.78524613.57816324.25673841.46385172.

42175863.86354712.51628347.35742186.27486531.14563278.78231654.63817425]

These examples show that Kochol's theorem is not best possible for $n \in\{5,6,7,8\}$ and hence leave wide open the question of what the best result might be in general. Cutler and Öhman [5] showed for all $m$ that every $(2 m k) \times(2 m k) \times m$ latin cuboid can be extended by 1 layer provided $k$ is sufficiently large.

\section{References}

[1] Y. Alavi, D. R. Lick and J. Liu, Strongly diagonal Latin squares and permutation cubes, Congr. Numer. 102 (1994) 65-70.

[2] R. A. Bailey, D. A. Preece and P. J. Zemroch, Totally symmetric Latin squares and cubes, Utilitas Math. 14 (1978), 161-170. 
[3] G. B. Beljavskaja and S. Murathudjaev, About admissibility of $N$-ary quasigroups, Combinatorics, Vol. I, pp. 101-119, Colloq. Math. Soc. János Bolyai, 18, NorthHolland, Amsterdam-New York, 1978.

[4] A. B. Cruse, On the finite completion of partial Latin cubes, J. Combin. Theory Ser. A 17 (1974), 112-119.

[5] J. Cutler and L.-D. Öhman, Latin squares with forbidden entries, Electron. J. Combin. 13 (2006), R47.

[6] J. Dénes and A. D. Keedwell, Latin squares and their applications, Akadémiai Kiadó, Budapest, 1974.

[7] N. J. Finizio and J. T. Lewis, Enumeration of maximal codes, Congr. Numer. 102 (1994), 139-145.

[8] H.-L. Fu, On Latin cubes with prescribed intersections, Ars Combin. 23 (1987), $171-176$.

[9] H. Gupta, On permutation cubes and Latin cubes, Indian J. Pure Appl. Math. 5 (1974), 1003-1021.

[10] M. Hall, An existence theorem for Latin squares, Bull. Amer. Math. Soc. 51 (1945), $387-388$.

[11] K. Heinrich, Prolongation in $m$-dimensional permutation cubes, Algebraic and geometric combinatorics, 229-238, North-Holland Math. Stud., 65, North-Holland, Amsterdam, 1982.

[12] T. Ito, Creation method of table, creation apparatus, creation program and program storage medium, US Patent application 20040243621, Dec. 2, 2004.

[13] X. W. Jia and Z. P. Qin, The number of Latin cubes and their isotopy classes, (Chinese) J. Huazhong Univ. Sci. Tech. 27, No. 11 (1999), 104-106.

[14] M. Kochol, Relatively narrow Latin parallelepipeds that cannot be extended to a Latin cube, Ars Combin. 40 (1995), 247-260.

[15] C.F. Laywine and G. L. Mullen, Latin cubes and hypercubes of prime order, Fibonacci Quart. 23 (1985), 139-145.

[16] C. F. Laywine and G. L. Mullen, Discrete Mathematics using Latin Squares, Wiley, New York, 1998.

[17] C. C. Lindner, A finite partial idempotent Latin cube can be embedded in a finite idempotent latin cube, J. Combin. Theory Ser. A 21 (1976), 104-109.

[18] B. D. McKay, A. Meynert and W. Myrvold, Small Latin squares, quasigroups and loops, J. Combin. Des., 15, (2007) 98-119.

[19] B. D. McKay, nauty graph isomorphic software, available at http://cs.anu.edu.au/ bdm/nauty. 
[20] B. D. McKay and I. M. Wanless, On the number of Latin squares, Ann. Comb. 9 (2005), 335-344.

[21] B. D. McKay and I. M. Wanless, Latin cubes and hypercubes, http://cs.anu.edu.au/ bdm/data/latincubes.html.

[22] G. L. Mullen and R. E. Weber, Latin cubes of order $\leq$ 5, Discrete Math. 32 (1980), 291-297.

[23] V.N. Potapov and D.S. Krotov, Asymptotics for the number of $n$-quasigroups of order 4, Siberian Math. J. 47 (2006), 720-731.

[24] D. A. Preece, S. C. Pearce and J. R. Kerr, Orthogonal designs for three-dimensional experiments, Biometrika 60 (1973), 349-358.

[25] N. J. A. Sloane, The On-Line Encyclopedia of Integer Sequences, http://www.research.att.com/ njas/sequences/.

[26] L. Teirlinck, Generalized idempotent orthogonal arrays, Coding theory and design theory, Part II, 368-378, IMA Vol. Math. Appl., 21, Springer, New York, 1990.

[27] H. S. Wilf, Generatingfunctionology (2nd edn.), Academic Press, San Diego, 1994.

[28] V.A. Zinoviev and D.Z. Zinoviev, Binary extended perfect codes of length 16 obtained by the generalized concatenated construction, Probl. Inf. Transm. 38 (2002), 296-322.

\section{Appendix}

In the following tables we present the number of paratopy classes of latin hypercubes $L$ with each combination of the parameters $d, n, I, P / I$, where $d$ is the dimension, $n$ is the order, $I=|\operatorname{Is}(L)|$ and $P=|\operatorname{Par}(L)|$. Lemma 1 explains how to compute the numbers of hypercubes, reduced hypercubes, and isotopy classes of hypercubes.

A representative of each of these paratopy classes can be found in [21]. 


\begin{tabular}{|c|c|c|c|c|c|c|c|c|c|c|c|c|}
\hline$d \quad n$ & $I$ & $P / I$ & count & d $\quad n$ & $I$ & $P / I$ & count & $d$ & $n$ & $I$ & $P / I$ & count \\
\hline \multirow[t]{2}{*}{3} & 8 & 24 & 1 & \multirow{38}{*}{$\begin{array}{ll}3 & 6\end{array}$} & 3 & 3 & 1 & 3 & 6 & 16 & 6 & 3 \\
\hline & \multicolumn{2}{|c|}{ total } & 1 & & 3 & 4 & 337 & & & 16 & 8 & 24 \\
\hline \multirow[t]{2}{*}{$\begin{array}{ll}3 & 3\end{array}$} & 54 & 24 & 1 & & 3 & 6 & 1 & & & 16 & 24 & 5 \\
\hline & \multicolumn{2}{|c|}{ total } & 1 & & 3 & 8 & 5 & & & 18 & 1 & 67 \\
\hline \multirow[t]{6}{*}{3} & 16 & 24 & 1 & & 4 & 1 & 14855 & & & 18 & 2 & 54 \\
\hline & 64 & 8 & 1 & & 4 & 2 & 3898 & & & 18 & 4 & 28 \\
\hline & 128 & 4 & 1 & & 4 & 3 & 1 & & & 18 & 6 & 2 \\
\hline & 128 & 24 & 1 & & 4 & 4 & 1121 & & & 18 & 8 & 10 \\
\hline & 384 & 24 & 1 & & 4 & 6 & 12 & & & 20 & 1 & 1 \\
\hline & \multicolumn{2}{|c|}{ total } & 5 & & 4 & 8 & 179 & & & 20 & 2 & 1 \\
\hline \multirow[t]{12}{*}{$\begin{array}{ll}3 & 5\end{array}$} & 3 & 4 & 5 & & 4 & 24 & 4 & & & 20 & 4 & 2 \\
\hline & 4 & 4 & 1 & & 5 & 4 & 1 & & & 24 & 1 & 67 \\
\hline & 4 & 24 & 1 & & 6 & 1 & 1302 & & & 24 & 2 & 75 \\
\hline & 6 & 24 & 1 & & 6 & 2 & 739 & & & 24 & 4 & 17 \\
\hline & 10 & 4 & 1 & & 6 & 4 & 244 & & & 24 & 6 & 1 \\
\hline & 10 & 8 & 1 & & 6 & 6 & 1 & & & 24 & 8 & 10 \\
\hline & 12 & 4 & 1 & & 6 & 8 & 13 & & & 27 & 1 & 5 \\
\hline & 20 & 24 & 1 & & 8 & 1 & 1118 & & & 27 & 2 & 11 \\
\hline & 60 & 24 & 1 & & 8 & 2 & 831 & & & 27 & 4 & 6 \\
\hline & 100 & 8 & 1 & & 8 & 3 & 1 & & & 27 & 8 & 3 \\
\hline & 500 & 24 & 1 & & 8 & 4 & 220 & & & 36 & 1 & 23 \\
\hline & \multicolumn{2}{|c|}{ total } & 15 & & 8 & 6 & 6 & & & 36 & 2 & 26 \\
\hline \multirow[t]{16}{*}{$\begin{array}{ll}33 & 6\end{array}$} & 1 & 1 & 75916 & & 8 & 8 & 65 & & & 36 & 4 & 8 \\
\hline & 1 & 2 & 17193 & & 9 & 1 & 137 & & & 36 & 6 & 1 \\
\hline & 1 & 3 & 29 & & 9 & 2 & 90 & & & 36 & 8 & 6 \\
\hline & 1 & 4 & 3049 & & 9 & 4 & 30 & & & 48 & 1 & 2 \\
\hline & 1 & 6 & 7 & & 9 & 6 & 1 & & & 48 & 2 & 9 \\
\hline & 1 & 8 & 309 & & 9 & 8 & 13 & & & 48 & 4 & 8 \\
\hline & 1 & 12 & 2 & & 10 & 2 & 1 & & & 48 & 6 & 1 \\
\hline & 1 & 24 & 3 & & 10 & 8 & 3 & & & 48 & 8 & 3 \\
\hline & 2 & 1 & 115256 & & 12 & 1 & 316 & & & 54 & 1 & 6 \\
\hline & 2 & 2 & 16921 & & 12 & 2 & 208 & & & 54 & 2 & 6 \\
\hline & 2 & 3 & 8 & & 12 & 4 & 53 & & & 54 & 4 & 5 \\
\hline & 2 & 4 & 2768 & & 12 & 6 & 1 & & & 54 & 8 & 4 \\
\hline & 2 & 6 & 15 & & 12 & 8 & 7 & & & 54 & 24 & 1 \\
\hline & 2 & 8 & 127 & & 16 & 1 & 22 & & & 72 & 1 & 9 \\
\hline & 3 & 1 & 4460 & & 16 & 2 & 65 & & & 72 & 2 & 14 \\
\hline & 3 & 2 & 1688 & & 16 & 4 & 28 & & & 72 & 4 & 4 \\
\hline
\end{tabular}

Table 3: Counts of paratopy classes by $I=|\operatorname{Is}(L)|$ and $P / I=|\operatorname{Par}(L)| /|\operatorname{Is}(L)|$ (part 1) 


\begin{tabular}{|c|c|c|c|c|c|c|c|c|c|c|c|c|}
\hline$d \quad n$ & $I$ & $P / I$ & count & $d \quad n$ & $I$ & $P / I$ & count & $d$ & $n$ & $I$ & $P / I$ & count \\
\hline \multirow{23}{*}{$\begin{array}{lr}3 & 6 \\
(\text { cont })\end{array}$} & 72 & 8 & 3 & \multirow[t]{7}{*}{$\begin{array}{ll}44 \\
\end{array}$} & 256 & 12 & 1 & \multirow{2}{*}{\multicolumn{2}{|c|}{52}} & 32 & 720 & 1 \\
\hline & 81 & 8 & 1 & & 512 & 8 & 2 & & & \multicolumn{2}{|c|}{ total } & 1 \\
\hline & 108 & 1 & 7 & & 512 & 10 & 1 & \multirow{2}{*}{\multicolumn{2}{|c|}{53}} & 486 & 720 & 1 \\
\hline & 108 & 2 & 2 & & 512 & 12 & 2 & & & \multicolumn{2}{|c|}{ total } & 1 \\
\hline & 108 & 4 & 1 & & 512 & 120 & 1 & \multirow{33}{*}{\multicolumn{2}{|c|}{54}} & 16 & 72 & 1 \\
\hline & 108 & 8 & 2 & & 1536 & 120 & 1 & & & 64 & 1 & 1909 \\
\hline & 120 & 2 & 1 & & \multicolumn{2}{|c|}{ total } & 26 & & & 64 & 2 & 1574 \\
\hline & 120 & 8 & 1 & \multirow[t]{30}{*}{45} & 1 & 1 & 3 & & & 64 & 3 & 9 \\
\hline & 144 & 2 & 3 & & 1 & 2 & 5 & & & 64 & 4 & 572 \\
\hline & 144 & 4 & 2 & & 1 & 6 & 3 & & & 64 & 6 & 61 \\
\hline & 144 & 8 & 3 & & 2 & 2 & 2 & & & 64 & 8 & 164 \\
\hline & 162 & 2 & 1 & & 2 & 4 & 1 & & & 64 & 10 & 9 \\
\hline & 216 & 1 & 2 & & 2 & 6 & 1 & & & 64 & 12 & 88 \\
\hline & 216 & 2 & 2 & & 2 & 12 & 1 & & & 64 & 16 & 51 \\
\hline & 216 & 8 & 1 & & 3 & 1 & 24 & & & 64 & 20 & 1 \\
\hline & 324 & 2 & 1 & & 3 & 2 & 7 & & & 64 & 24 & 5 \\
\hline & 324 & 4 & 1 & & 3 & 6 & 3 & & & 64 & 36 & 8 \\
\hline & 432 & 2 & 1 & & 4 & 6 & 1 & & & 64 & 48 & 22 \\
\hline & 432 & 24 & 1 & & 5 & 2 & 9 & & & 64 & 60 & 1 \\
\hline & 648 & 2 & 1 & & 5 & 4 & 4 & & & 64 & 72 & 5 \\
\hline & 720 & 8 & 1 & & 10 & 2 & 1 & & & 64 & 120 & 4 \\
\hline & 1296 & 8 & 1 & & 10 & 4 & 2 & & & 64 & 720 & 3 \\
\hline & \multicolumn{2}{|c|}{ total } & 264248 & & 10 & 6 & 2 & & & 128 & 1 & 16 \\
\hline \multirow[t]{2}{*}{42} & 16 & 120 & 1 & & 10 & 12 & 1 & & & 128 & 2 & 24 \\
\hline & \multicolumn{2}{|c|}{ total } & 1 & & 12 & 2 & 1 & & & 128 & 4 & 54 \\
\hline \multirow[t]{2}{*}{43} & 162 & 120 & 1 & & 12 & 6 & 1 & & & 128 & 6 & 8 \\
\hline & \multicolumn{2}{|c|}{ total } & 1 & & 20 & 2 & 2 & & & 128 & 8 & 13 \\
\hline \multirow[t]{10}{*}{$4 \quad 4$} & 32 & 8 & 2 & & 20 & 12 & 1 & & & 128 & 12 & 15 \\
\hline & 32 & 10 & 1 & & 25 & 8 & 2 & & & 128 & 16 & 12 \\
\hline & 32 & 12 & 3 & & 50 & 4 & 1 & & & 128 & 36 & 2 \\
\hline & 32 & 120 & 2 & & 50 & 8 & 2 & & & 128 & 48 & 14 \\
\hline & 64 & 4 & 3 & & 50 & 12 & 2 & & & 128 & 60 & 1 \\
\hline & 64 & 12 & 2 & & 100 & 8 & 1 & & & 128 & 720 & 2 \\
\hline & 64 & 24 & 2 & & 100 & 12 & 1 & & & 256 & 1 & 4 \\
\hline & 128 & 8 & 1 & & 500 & 12 & 1 & & & 256 & 2 & 26 \\
\hline & 256 & 4 & 1 & & 2500 & 120 & 1 & & & 256 & 4 & 24 \\
\hline & 256 & 8 & 1 & & \multicolumn{2}{|c|}{ total } & 86 & & & 256 & 6 & 8 \\
\hline
\end{tabular}

Table 4: Counts of paratopy classes by $I=|\operatorname{Is}(L)|$ and $P / I=|\operatorname{Par}(L)| /|\operatorname{Is}(L)|$ (part 2) 


\begin{tabular}{|c|c|c|c|c|c|c|c|c|c|c|c|c|}
\hline$d \quad n$ & $I$ & $P / I$ & count & $d$ & $n$ & $I$ & $P / I$ & count & d $\quad n$ & $I$ & $P / I$ & count \\
\hline \multirow{24}{*}{$\begin{array}{lr}5 & 4 \\
\text { (cont) }\end{array}$} & 256 & 8 & 18 & \multirow{37}{*}{\multicolumn{2}{|c|}{55}} & 2 & 8 & 1 & \multirow[t]{35}{*}{$\begin{array}{ll}5 & 5\end{array}$} & 25 & 6 & 9 \\
\hline & 256 & 12 & 6 & & & 2 & 9 & 1 & & 25 & 8 & 81 \\
\hline & 256 & 16 & 6 & & & 2 & 12 & 3 & & 25 & 12 & 4 \\
\hline & 256 & 24 & 1 & & & 2 & 18 & 10 & & 25 & 16 & 5 \\
\hline & 256 & 36 & 2 & & & 2 & 36 & 6 & & 50 & 4 & 4 \\
\hline & 256 & 48 & 7 & & & 3 & 1 & 1128 & & 50 & 6 & 1 \\
\hline & 512 & 4 & 2 & & & 3 & 2 & 378 & & 50 & 8 & 13 \\
\hline & 512 & 12 & 1 & & & 3 & 3 & 31 & & 50 & 12 & 3 \\
\hline & 512 & 16 & 2 & & & 3 & 4 & 55 & & 50 & 16 & 5 \\
\hline & 1024 & 4 & 3 & & & 3 & 6 & 39 & & 50 & 18 & 2 \\
\hline & 1024 & 8 & 2 & & & 4 & 2 & 2 & & 50 & 36 & 1 \\
\hline & 1024 & 12 & 3 & & & 4 & 3 & 1 & & 60 & 36 & 1 \\
\hline & 1024 & 16 & 3 & & & 4 & 4 & 1 & & 100 & 4 & 3 \\
\hline & 1024 & 48 & 2 & & & 4 & 6 & 2 & & 100 & 6 & 2 \\
\hline & 1024 & 72 & 1 & & & 4 & 18 & 3 & & 100 & 8 & 8 \\
\hline & 2048 & 4 & 4 & & & 4 & 36 & 3 & & 100 & 12 & 1 \\
\hline & 2048 & 12 & 2 & & & 4 & 72 & 1 & & 100 & 16 & 1 \\
\hline & 2048 & 16 & 2 & & & 5 & 2 & 468 & & 100 & 36 & 1 \\
\hline & 2048 & 36 & 1 & & & 5 & 4 & 18 & & 125 & 8 & 2 \\
\hline & 2048 & 48 & 4 & & & 5 & 6 & 26 & & 125 & 12 & 2 \\
\hline & 2048 & 60 & 1 & & & 5 & 12 & 2 & & 125 & 24 & 2 \\
\hline & 2048 & 720 & 1 & & & 6 & 18 & 2 & & 125 & 48 & 1 \\
\hline & 6144 & 720 & 1 & & & 6 & 36 & 3 & & 250 & 8 & 1 \\
\hline & \multicolumn{2}{|c|}{ total } & 4785 & & & 10 & 2 & 14 & & 250 & 12 & 3 \\
\hline \multirow[t]{13}{*}{55} & 1 & 1 & 337 & & & 10 & 4 & 14 & & 250 & 16 & 3 \\
\hline & 1 & 2 & 105 & & & 10 & 6 & 10 & & 250 & 36 & 1 \\
\hline & 1 & 3 & 72 & & & 10 & 8 & 4 & & 250 & 48 & 2 \\
\hline & 1 & 4 & 28 & & & 10 & 12 & 5 & & 500 & 12 & 1 \\
\hline & 1 & 6 & 40 & & & 10 & 36 & 1 & & 500 & 16 & 2 \\
\hline & 1 & 12 & 4 & & & 12 & 2 & 2 & & 500 & 36 & 1 \\
\hline & 1 & 18 & 3 & & & 12 & 3 & 1 & & 500 & 48 & 1 \\
\hline & 1 & 36 & 4 & & & 12 & 4 & 1 & & 2500 & 48 & 1 \\
\hline & 2 & 1 & 3 & & & 12 & 6 & 1 & & 2500 & 72 & 1 \\
\hline & 2 & 2 & 6 & & & 20 & 2 & 2 & & 12500 & 720 & 1 \\
\hline & 2 & 3 & 7 & & & 20 & 12 & 1 & & tot: & & 3102 \\
\hline & 2 & 4 & 7 & & & 20 & 72 & 1 & & & & \\
\hline & 2 & 6 & 5 & & & 25 & 4 & 70 & & & & \\
\hline
\end{tabular}

Table 5: Counts of paratopy classes by $I=|\operatorname{Is}(L)|$ and $P / I=|\operatorname{Par}(L)| /|\operatorname{Is}(L)|$ (part 3) 